\title{
A Roomy Survey on Content Based Image Retrieval
}

\author{
Dr. Ruksar Fatima ${ }^{1}$, Prof. Feroza. D. Mirajkar ${ }^{2}$, Prof.Vinay C Warad ${ }^{3}$, Prof. Sameena \\ Sarwath $^{4}$ \\ 1 vice-principal and head of Department of computer science and Engineering \\ 2,Department of Electronics and communication Engineering \\ 3,Department of Computer Science and Engineering, \\ 4 Department of Electronics and instrumentation Engineering, \\ 1,2,3,4 Khaja Banda Nawaz of Engineering, Kalaburagi
}

\section{Abstract:}

The extent of images or the pictorial evidence is growing day by day with the expansion of internet services. As the network and development of multimedia technologies are becoming more attractive, users are not contented with the traditional information retrieval techniques. So nowadays the content based image retrieval is becoming a dawn of exact and fast retrieval. It is very difficult for the users to retrieve the required images using a usable and efficient Contrivance. There are many techniques which are used to retrieve the images depending upon the prerequisite of different applications. This paper provides an roomy review of various latest research work and methodologies applied in the field of CBIR. Images are retrieved on the basis of automatically derived features such as, shape, color and texture which is generally referred to as Content-Based Image Retrieval (CBIR). Content based image retrieval is an interesting research area in image processing, with a vast domain of applications like recognition systems i.e. finger, face, biometrics, medical sciences etc. However, the technology still has scarcity of maturity, and is not yet being used on a momentous scale. In the truancy of hard substantiation on the effectiveness of CBIR techniques in practice, judgment is still sharply divided about their usefulness in handling real-life queries in large and diverse image collections.

\section{Keywords - Region Based Image Retrieval (RBIR), Subspace learning based Techniques, CBIR}

\section{INTRODUCTION}

CBIR (Content based image retrieval) is the software system for retrieving the images from the database by using their features. In CBIR technique, the images are retrieved from the dataset by using the features like color, text, shape, texture and similarity. Object recognition technique is used in CBIR. Research on multimedia systems and content-based image retrieval is given tremendous importance during the last decade. The reason behind this is the fact that multimedia databases deal with text, audio, video and image data, which are of prime interest in web and other high end user applications. Content-based Image retrieval deals with the extraction of knowledge, image data relationship, or other patterns not explicitly stored in the images. It uses methods from computer vision, image processing, image retrieval, data retrieval, machine learning, database and artificial intelligence. Rule retrieval has been applied to large image databases.

A vast amount of image data such as satellite images, medical images, and digital photographs, if analyzed can reveal useful information to the human users. Unfortunately, it is difficult or even 
impossible for human to discover the underlying knowledge and patterns in the image when handling a large collection of images. It is rapidly gaining attention among researchers in the field of data retrieval, information retrieval, and multimedia databases because of its potential in discovering useful image patterns. The fundamental challenge in image retrieval is to determine how low-level, pixel representation contained in a raw image or image sequence can be efficiently and effectively, can be processed to identify high-level spatial objects and relationships.

Research in image retrieval can be broadly classified into two main directions. The first direction involves domain-specific applications where the focus is to extract the most relevant image features into a form suitable for data retrieval. The second direction involves general applications where the focus is to generate image patterns that may be helpful in the understanding of the interaction between high-level human perceptions of images and low-level image features. Effective and operative retrieval of images from a large data base is a very difficult task. Therefore the retrieval of similar and relevant images based on the similarity between automatically derived content features such as color shape, texture, etc of the query image and that of the images which are stored in the data base and that task is popularly known as content based image retrieval. The term color can be achieved by the techniques histogram and averaging [18]. The term texture refers the use of vector quantization or transforms. The term shape is the use of gradient operators or morphological operators [18]. The accuracy of the CBIR system can be improved by the iterative refinement process of the queries and the features that are decided by the user's feedback [19].

Image indexing with suitable and appropriate features has become an important research topic recently .Indexing is the characterization of the images based on one or more image features or properties [17] .An image consists of global and local features. Depending upon the problem we can use the features of our interest to retrieve the images from a database [17].

Some of the major areas of applications of CBIR are: medical diagnosis, Intellectual property, art collections, crime prevention, military and engineering design and geographical information and remote sensing systems.

The steps that are to be followed in the system realization of CBIR are [16]:

1. Image acquisition

2. Feature Extraction

3. Similarity Matching

A large number of images are stored in the database. Image enhancement takes place where various techniques are applied on the image to improve its quality like histogram manipulation .The enhanced image is then subdivided and segmented to get the color, texture and edge density features forming a feature vector. The resultant feature vector can be compared with the feature vector of the query image [19]. The closest image in comparison with the query image from the feature database is returned.

Pictures are the most common and convenient means of conveying information. A picture is worth thousand words. The overall objective of image classification is to automatically categorize all pixels in an image. Hence the core of the work is efficient implementation of generalized or specific pattern recognition techniques. We developed the software system for retrieving the images from the database by using their features in image retrieving technique and finally making the comparison of all the image retrieval techniques. 


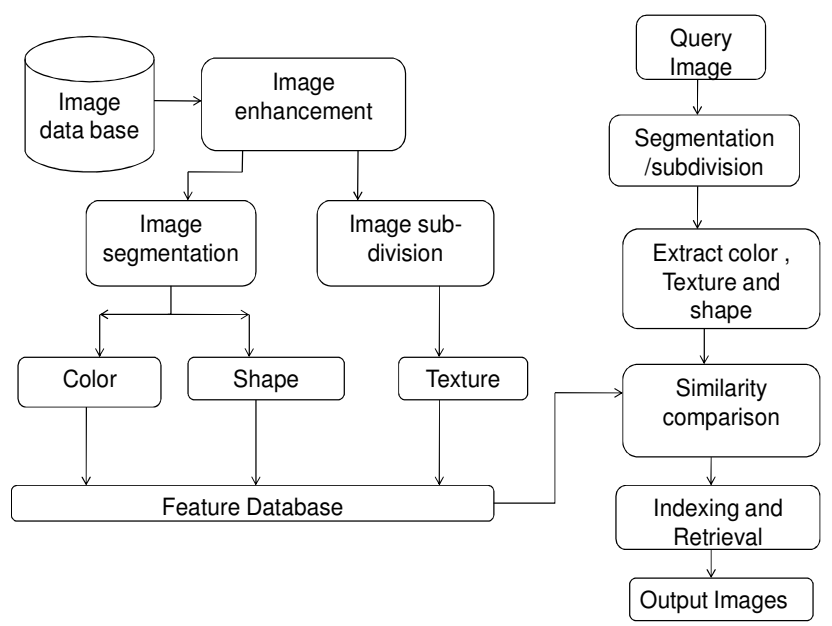

Figure 1: Block diagram for Content-Based Image Retrieval

\section{Region Based Image Retrieval (RBIR)}

Early CBIR methods used global feature extraction to obtain the image descriptors. For example, QBIC developed at the IBM Almaden Research Center extracts several features from each image, namely color, texture, and shape features. These descriptors are obtained globally by extracting information by means of color histograms for color features; global texture information on coarseness, contrast, and direction; and shape features about the curvature, moments invariants, circularity, and eccentricity. Similarly, the Photo book system, Visual seek, and VIR, use global features to represent image semantics.

These global approaches are not adequate to support queries looking for images where specific objects in an image with particular colors and/or texture are present, and shift/scale invariant queries, where the position and/or the dimension of the query objects may not be relevant. For example, suppose in one image there are two flowers with different colors: red and yellow. The global features describe the image as the average of the global average color which is orange. This description is certainly not there presentation of the semantic meaning of the image. Therefore, the weakness of global features is observable.
Region-based retrieval systems attempt to overcome previous method limitations of global based retrieval systems by representing images as collections of regions that may correspond to objects such as flowers, trees, skies and mountains . A key prerequisite for a good region based image retrieval system is a robust segmentation algorithm. A segmentation algorithm takes an input image and clusters pixels of this image that seem to be similar with respect to some feature (e.g. color, texture, or shape). The result of this clustering phase is to decompose an image into regions, which correspond to physical objects (trees, people, cars, flowers) if the decomposition is ideal. The feature descriptors are then extracted from each object instead of global image. Color, texture, and shape features are extracted on each pixel that belongs to the object, and each object is described by the average value of these pixel features.

For efficient image retrieval we have discussed feature based challenges in the previous section, in this section we discuss the major challenges in terms of classifiers.

SVM RF approaches ignore the basic difference between the two distinct groups of feedbacks i.e., all positive feedbacks share a similar concept while each negative feedback usually varies. This has been found to drastically degrade the effectiveness of this method. This can be overcome by implementing CBIR both on-line and off-line. Also choosing proper kernel functions and parameters for a real specific database remains challenging. The number of support vectors that compose the decision function increase dramatically when the decision procedure becomes complicated. Moreover the over fitting problem can become more severe i.e., training samples may be few to train a good classifier in a high dimensional space. 
International Journal of Engineering and Techniques -Volume 4, Issue 5, Sept - Oct 2018

\section{LITERATURE SURVEY}

1. L Zhang, L Wang and Weisi Lin" A Semantic Subspace Learning Method to Exploit Relevance Feedback Log Data for Image Retrieval", 2013 IEEE [1]

Method employed: Semantic subspace learning from RF log data with contextual data without using any class label information.

Results obtained/ merits: RF has been introduced to improve performance of CBIR, No class label information, Cannot achieve satisfactory performance due to its semantic gap.

Limitations: learning from small training sets

2. Roshi Choudhary, Nikita Raina, Neeshu Chaudhary, Rashmi Chauhan, Dr. $R H$ Goudar," An Integrated Approach to Content Based Image Retrieval”, 978-14799-3080-7114/\$31.00 @2014 IEEE [2]

Method employed: Integrated approach (color, texture), Both color and texture feature of image are combined to form a single feature vector.

Merits :More accuracy rather than only color and texture, Color moment.

Limitations : Global feature for color

3. L. Jiao, X. Tang, B. Hou and S. Wang, "SAR Images Retrieval Based on Semantic Classification and Region-Based Similarity Measure for Earth Observation," in IEEE Journal of Selected Topics in Applied Earth Observations and Remote Sensing, vol. 8, no. 8, pp. 3876-3891, Aug. 2015.[3]

Method employed: IIRM- improved integrated region matching, measured based on region based similarity measure, SSLclassic semi-supervised learning, decrease searching space in similarity calculating component .

Merits : Provides Error recovery scheme by "empirical confusion matrix"

Limitations : Scheme of recovery is hard threshold approach which is simple but unfair sometimes

4. S. R. Dubey, S. K. Singh and R. Kumar Singh, "Local neighbourhood-based robust colour occurrence descriptor for colour image retrieval," in IET Image Processing, vol. 9, no. 7, pp. 578-586, 72015 [4]

Methods employed: Quantization of RGB space- number of colors are reduced into a less number of shades, Local color occurrence binary pattern is generated for each pixel of image by representing each reduced color shade cooccurence in its local neighbourhood using binary pattern.

Merits: Capture more relevant local color information of each quantized color shade

Limitations: Time consuming - RGB space is quantized in 64 shades to represent the feature of image.

5. J. Luo, Z. Jiang and J. Li, "Multi-scale order less cross-regions-pooling of deep attributes for image retrieval," in Electronics Letters, vol. 52, no. 4, pp. 276277, 218 2016.[5]

Methods employed: Cross region pooling(CRP) is build combining two key ingredients, region proposals detected by objectness detection technique, deep attributes (DA) i.e, output of softmax layer of off-the-shelf convolution neural network pertained on a large scale data set. 
International Journal of Engineering and Techniques -Volume 4, Issue 5, Sept - Oct 2018

Merits: Regions may be well aligned with context in images. DA's(deep attributes) are probability responses to categories on which CNN's are trained and hence are fairly semantic, CRP of DA is robust to noisy regions and surpass redundant regions, result is robust image representation.

Limitations: Region feature extraction through Time consuming $(\mathrm{CNN})$ is time consuming

6. Chad Carson and Virginia E. Ogle. "Storage and retrieval of feature data for a very large online image collection". IEEE Computer Society Bulletin of the Technical Committee on Data Engineering, 19(4):19_ 27, December 1996.[6]

Methods employed: Region histogram.

Merits: Data is stored in such a way as to facilitate the fastest possible retrieval time in order to make rapid online browsing feature, Features are stored as text field.-for fast retrieval and incremental changes to stored feature data do not require elaborate reencoding or new attribute names.

Limitations: Mismatch in the results of the retrieval process when the orientation, position or scales of images are altered

7. M. Stricker and M. Orengo. "Similarity of color images". In W. Niblack and R. Jain, editors, Storage and Retrieval for Image and Vid eo DatabasesIII (SPIE), volume 2420, pages 381\{392, San Diego/La Jolla, CA, USA, February 1995.[7]

Methods employed: Color moment, First method - use cumulative color histogram, Second method- color indexing.

Merits: First method - More robust than with respect to quantization parameters of histograms and color histogram indexing and results are slightly better than color histograms Color indexing-stores complete color distribution and only dominant features

Limitations: Semantically relevant images will be retrieved with amount of time

8. Pentland A et al (1996) "Photobook: tools for contentbased manipulation of image databases" International Journal of Computer Vision 18(3), 233254[8]

Methods employed: Elastic deformation of templates, "wold transform " for decomposing signals into compact, "Karhunen-Loeve transform" to derive optimally compact represented for appearance or shape.

Merits: Semantics preserving image compression which reduces images to a small set of perceptually significant co-efficients, Provides users with a sophisticated and efficient utility for database search based on image content.

Limitations: The similarity distance is found between the query image and the images belonging to the predicted cluster alone.

9. J. R. Smith and S. F. Chang, "Transform features for texture classification and discrimination in large image databases", in Proc. IEEE Int. Conf. on Image Proc., 1994[9]

Methods employed: Wavelet transform, A large image database from Brodatz album is used for retrieval purpose.

Merits: Canberra Bray-Curtis, Square chord and Square Chi-Squared distances are superior over the conventional Euclidean and Manhattan distance. 
International Journal of Engineering and Techniques -Volume 4, Issue 5, Sept - Oct 2018

Limitations: All the classes are assigned to one of the two possible groups of classes

10. B. S. Manjunath and W. Y. Ma. "Texture features for browsing and retrieval of large image data” IEEE Transactions on Pattern Analysis and Machine Intelligence, (Special Issue on Digital Libra ries), Vol. 18 (8), August 1996, pp. 837842.[10]

Methods employed: Gabor filters, Brodatz texture database and Adaptive filter selection strategy

Merits: Gabor wavelet features for texture analysis are used to provide a comprehensive experimental evaluation, the Gabor features provide the best pattern retrieval accuracy, a novel adaptive filter selection strategy is suggested to reduce the image processing computations and gabor filter is quiet robust.

Limitations: The machine learning predicts the category of the query image

\section{George Tzagkarakis and Panagiotis} Tsakalides. "A Statistical approach to texture image retrieval via alphastable modeling of wavelet decompositions". in Proc. 5th International Workshop on Image Analysis for Multimedia Interactive Services (WIAMIS 04), Lisbon, Portugal, April 2123, 2004 [11]

Methods employed: statistical and retrieval scheme is first applied on one-dimensional synthetic data, and then on a set of texture images found in the VisTex data base and wavelet-based texture retrieval method is introduced by computing Kullback-Leibler distance measures between alpha stable distributions.

Merits: Scheme can be used successfully when the data follow algebraic tails.
Limitations: The query image belongs to the class for which the membership is very large

12. S.F. Chang, W. Chen, H. Sundaram, "Semantic visual templates: linking visual features to semantics", International Conference on Image Processing (ICIP), Workshop on Content Based Video Search and Retrieval, vol. 3, October 1998, pp. 531534. [12]

Methods employed: Semantic visual template is derived bridging the gap between the low-level features that are derived from the raw data to semantics.

Merits: This system performs well, for example with small number of queries in the "sunset" template, we are able to achieve $50 \%$ recall and $24 \%$ precision over a large unannotated database, SVT's provides a mechanism for a two-way interaction between the user and the system and the user can compose a new concept by using preexisting library of templates.

Limitations: Texture descriptors contain features derived from co-occurrence matrices

\section{Janghyun Yoon and Nikil Jayant. "Relevance Feedback for semantics based Image retrieval” [13]}

Methods employed: Multimedia model feedback and Relevance feedback.

Merits: This approach makes image retrieval more semantics-sensitive and improves the image retrieval performance significantly.

Limitations: Descriptors based on color representation might be effective with a data set containing black and white images

14. H. Feng and T.S. Chua. "A bootstrapping approach to annotating large image 
International Journal of Engineering and Techniques -Volume 4, Issue 5, Sept - Oct 2018

collection." Proceedings of the Fifth ACM SIGMM international workshop on Multimedia information retrieval (MIR 03), pages 55\{62, Berkeley, California, USA, November 2003.[14]

Methods employed: Automatic and Boot strapping.

Merits: A co-training approach that fuses evidences from image contents and their associated HTML text is developed and the co-training approach could achieve a level of performance comparable to that of the supervised learning approach but requiring a much smaller set of labeled training samples is being demonstrated.

Limitations: Statistical approaches do not exploit the sensitivity of the human visual system to textures.

15. P.L. Stanchev, D. Green Jr., B. Dimitrov, "High level color similarity retrieval", Int. J. Inf. Theories Appl. 10 (3) (2003) 363369[15]

Methods employed: Object ontology and Color representation ontology.

Merits: Spatiogram, a generalization of histogram to higher-order moments, more efficient for content based retrieval of remote sensing images and efficiency of the system can be increased by increasing the number of quantization bins at small cost of time.

Limitations: It is extremely difficult to describe high level semantic concepts with image features only.

\section{CONCLUSION}

This paper provides an rundown of the performance of content based image retrieval systems. Most systems use color and texture features, few systems use shape feature, and still less use layout features. It also discusses about various feature extraction methods, similarity measurement techniques and the various applications. It has been found that variation in feature extraction methodologies can ensure the better and more accurate retrieval of relevant images from the large database. The CBIR system also depends on the size of the database.

\section{References}

[1] L Zhang, L Wang and Weisi Lin" A Semantic Subspace Learning Method to Exploit Relevance Feedback Log Data for Image Retrieval”, 2013 IEEE

[2] Roshi Choudhary, Nikita Raina, Neeshu Chaudhary, Rashmi Chauhan, Dr. R H Goudar," An Integrated Approach to Content Based Image Retrieval”, 978-14799-3080-7114/\$31.00 @2014 IEEE

[3] L. Jiao, X. Tang, B. Hou and S. Wang, "SAR Images Retrieval Based on Semantic Classification and Region-Based Similarity Measure for Earth Observation," in IEEE Journal of Selected Topics in Applied Earth Observations and Remote Sensing, vol. 8, no. 8, pp. 3876-3891, Aug. 2015.

[4] S. R. Dubey, S. K. Singh and R. Kumar Singh, "Local neighbourhood-based robust colour occurrence descriptor for colour image retrieval," in IET Image Processing, vol. 9, no. 7, pp. 578-586, 72015

[5] J. Luo, Z. Jiang and J. Li, "Multi-scale order less cross-regions-pooling of deep attributes for image retrieval," in Electronics Letters, vol. 52, no. 4, pp. 276-277, 2182016.

[6] Chad Carson and Virginia E. Ogle. "Storage and retrieval of feature data for a very large online image collection”. IEEE Computer Society Bulletin of the Technical Committee on Data Engineering, 19(4):1927, December 1996.

[7] M. Stricker and M. Orengo. "Similarity of color images”. In W. Niblack and R. Jain, editors, Storage and Retrieval for Image and Vid eo DatabasesIII (SPIE), volume 2420, pages 381\{392, San Diego/La Jolla, CA, USA, February 1995.

[8] Pentland A et al (1996) "Photobook: tools for contentbased manipulation of image databases" International Journal of Computer Vision 18(3), 233254

[9] J. R. Smith and S. F. Chang, "Transform features for texture classification and discrimination in large image databases", in Proc. IEEE Int. Conf. on Image Proc., 1994 
International Journal of Engineering and Techniques -Volume 4, Issue 5, Sept - Oct 2018

[10]B. S. Manjunath and W. Y. Ma. "Texture features for browsing and retrieval of large image data" IEEE Transactions on Pattern Analysis and Machine Intelligence, (Special Issue on Digital Libra ries), Vol. 18 (8), August 1996, pp. 837842.

[11]George Tzagkarakis and Panagiotis Tsakalides. "A Statistical approach to texture image retrieval via alphastable modeling of wavelet decompositions". in Proc. 5th International Workshop on Image Analysis for Multimedia Interactive Services (WIAMIS 04), Lisbon, Portugal, April 2123, 2004

[12]S.F. Chang, W. Chen, H. Sundaram, "Semantic visual templates: linking visual features to semantics", International Conference on Image Processing (ICIP), Workshop on Content Based Video Search and Retrieval, vol. 3, October 1998, pp. 531-534.

[17]Minakshi Banerjee Malay. K. Kundu, "Content Based Image Retrieval with Multiresolution Salient Points Machine Intelligence Unit”, Kolkata 700108, INDIA

[18] "Mean and standard deviation features of color histogram using Laplacian filter for image retrieval”,
[13]Janghyun Yoon and Nikil Jayant. "Relevance Feedback for semantics based Image retrieval"

[14]H. Feng and T.S. Chua. "A bootstrapping approach to annotating large image collection." Proceedings of the Fifth ACM SIGMM international workshop on Multimedia information retrieval (MIR 03), pages 55\{62, Berkeley, California, USA, November 2003.

[15]P.L. Stanchev, D. Green Jr., B. Dimitrov, "High level color similarity retrieval”, Int. J. Inf. Theories Appl. 10 (3) (2003) 363-369

[16]Dr. H.B. Kekre et al, "A survey of CBIR techniques and semantics", International Journal of Engineering Science and Technology (IJEST),Vol. 3 No. 5,45104517, May 2011

Journal of Theoretical and Applied Information Technology,1-7, Vol. 34 No.1, 15th December 2011

[19]Nidhi Singhai, Shishir K. Shandily, "A survey on content Based image retrieval systems" , International Journal of Computer Applications (0975 - 8887),2226, Volume 4 - No.2, July 2010, India 